\title{
Prevalência de adenomas colorretais em pacientes com história familiar para câncer colorretal
}

\author{
Prevalence of colorectal adenomas in patients with family history of colorectal cancer.
}

\author{
BIANCA ZANDONÁ ${ }^{1}$, LUCIANO PINTO DE CARVALHO ${ }^{2}$, JULIA SCHIMEDT ${ }^{3}$, DANIELA CERQUEIRA KOPPE ${ }^{4}$, \\ RUY TAKASHI KOSHIMIZU ${ }^{4}$, AFONSO CALIL MURY MALLMANN ${ }^{4}$
}

${ }^{1}$ Acadêmica do Curso de Medicina da Universidade Luterana do Brasil (ULBRA), Campus Canoas - Canoas (RS), Brasil. ${ }^{2}$ Doutor em Medicina; Professor orientador e Professor adjunto do Curso de Medicina da ULBRA-Canoas (RS), Brasil; Médico do Serviço de Coloproctologia do Hospital Nossa Senhora da Conceição - Porto Alegre (RS), Brasil. ${ }^{3}$ Médica do Hospital Nossa Senhora da Conceição- Porto Alegre (RS), Brasil. ${ }^{4}$ Médico do Serviço de Coloproctologia do Hospital Nossa Senhora da Conceição - Porto Alegre (RS), Brasil.

ZANDONÁ B, CARVALHO LP, SCHIMEDT J, KOPPE DC, KOSHIMIZU RT, MALLMANN ACM. Prevalência de adenomas colorretais em pacientes com história familiar para câncer colorretal. Rev bras Coloproct, 2011;31(2): 147-154.

RESUMO: $O$ câncer colorretal (CCR) é uma doença comum e letal, influenciada por fatores genéticos e ambientais, e pela interação entre ambos. Seu risco está fortemente associado ao número e à idade dos parentes de primeiro grau com história de CCR. O objetivo foi estudar a prevalência de adenomas em pacientes com história familiar de câncer colorretal. Métodos: Realizou-se um estudo retrospectivo, tendo como alvo todos os pacientes submetidos à colonoscopia em um hospital de referência da cidade de Porto Alegre, entre 2003 e 2007. Resultados: Dos 2.462 pacientes estudados, 118 apresentaram história familiar para CCR e 2.344 não apresentaram. Nos dois grupos, houve predomínio do sexo feminino $(\mathrm{P}=0,001)$. Nos pacientes com adenomas e história familiar para $\mathrm{CCR}$, houve predomínio do sexo feminino. Já nos pacientes com adenomas e sem história familiar, o sexo masculino foi mais frequente $(\mathrm{P}=0,032)$. $O$ número de adenomas entre eles não foi estatisticamente significativo $(\mathrm{P}=\mathbf{0 , 1 8 7})$. Conclusão: $O$ grupo com história familiar para CCR mostrou mais mulheres $(\mathrm{P}=\mathbf{0 , 0 0 1}) \mathrm{e}$ a idade também foi inferior $(\mathrm{P}=\mathbf{0 , 0 0 2})$. A comparação entre o grupo com adenomas e história familiar para CCR mostrou um número maior de mulheres que o grupo com adenomas sem história familiar para CCR (P=0,032).

Palavras-chave: adenoma; prevalência; câncer colorretal.

\section{INTRODUÇÃO}

O câncer colorretal é um dos tumores sólidos mais comuns, sendo considerado o segundo mais prevalente no mundo e a terceira neoplasia mais diagnosticada nos Estados Unidos. É responsável por aproximadamente $10 \%$ dos óbitos por câncer nos países ocidentais, representando, nessa região, a segunda causa de morte oncológica, perdendo apenas para o câncer de pulmão ${ }^{1}$. No Brasil, os tumores de cólon e reto estão entre as seis neoplasias mais prevalentes entre o sexo masculino. Já, no sexo feminino é o terceiro mais frequente ${ }^{2}$.
Pólipo colorretal é definido como qualquer projeção ou elevação da superfície da mucosa colorretal, podendo originar-se de qualquer camada da parede intestinal ${ }^{3}$. São divididos, de acordo com a sua histologia, em neoplásicos, hamartomatosos, inflamatórios e hiperplásicos. O mais frequente e mais importante pólipo colorretal é o adenoma: uma neoplasia benigna do epitélio.

O desenvolvimento do câncer colorretal ocorre pelo resultado da transformação do epitélio colônico normal em lesões benignas, denominadas pólipos adenomatosos, que posteriormente evoluem para lesões malignas, os adenocarcinomas ${ }^{4}$. A progressão é lenta,

Trabalho realizado no Hospital Nossa Senhora da Conceição, Porto Alegre (RS), Brasil.

Fonte de financiamento: não há

Conflito de interesse: nada a declarar

Recebido em: $24 / 05 / 2011$

Aprovado em: 06/07/2011 
geralmente envolvendo vários anos e com um número considerável de alterações genéticas ${ }^{5}$.

Os adenomas colorretais, devido ao potencial de malignização, possuem um importante significado clínico; porém, não é possível apontar quais desses evoluirão para adenocarcinomas. Há, entretanto, uma classificação para os adenomas baseada em seu grau de displasia, sendo determinada pela atipia celular, ordenamento das células para a formação de túbulos e figuras de mitose, tamanho nuclear e hipercromasia nuclear. Dessa forma, são divididos em baixo, indeterminado e alto, indicando graus progressivos ${ }^{6,7}$.

Alguns adenomas são considerados de alto risco, levando em consideração a histologia vilosa do pólipo, o tamanho superior a um centímetro, a presença de três ou mais adenomas e a displasia de alto grau. Esses fatores, por sua vez, são independentes para o desenvolvimento do câncer ${ }^{8}$.

Aproximadamente $75 \%$ dos adenomas colorretais são ditos esporádicos, significando ausência de características de hereditariedade ou de doenças predisponentes; todavia, acredita-se que a hereditariedade seja responsável por cerca de 3 a 5\% dos casos de câncer colorretal $^{9,10}$. O risco da doença maligna em famílias com esse tipo de neoplasia já está bem caracterizado; porém, tal afirmação não se mostra concreta em famílias de pacientes com pólipos adenomatosos ${ }^{11}$.

Indivíduos com antecedentes de remoção de adenomas apresentam maior risco de desenvolver novos adenomas do que a população em geral. Segundo estudos, a incidência de novos adenomas após um ano de ressecção inicial é de $30 \%$, sendo mais elevada nos pacientes com maior número de adenomas no primeiro exame ${ }^{12}$.

O seguimento colonoscópico é recomendado a todos os pacientes portadores de adenomas. Há, portanto, uma necessidade de realização de mais estudos a fim de confirmar, de maneira mais adequada, o intervalo de tempo ideal para a realização de novas colonoscopias nos pacientes submetidos à polipectomias, principalmente os que apresentam história familiar ou pessoal positiva para câncer colorretal.

Devido a isso, o objetivo deste trabalho foi estudar a prevalência de pólipos adenomatosos em pacientes, cuja indicação do exame de colonoscopia deveu-se ao histórico familiar de câncer colorretal, e comparar com os pólipos adenomatosos de pacientes sem histórico familiar para câncer colorretal, em um hospital de referência de Porto Alegre.

\section{MÉTODOS}

Realizou-se um estudo retrospectivo, tendo como alvo todos os pacientes submetidos a colonoscopias, no Serviço de Coloproctologia do Hospital Nossa Senhora da Conceição em Porto Alegre (RS), de 2003 a 2007.

Os critérios de inclusão da amostra foram todos os pacientes submetidos ao exame de colonoscopia. Os de exclusão foram: pacientes com pólipos que não fossem adenomatosos e também os pacientes que apresentassem polipose adenomatosa familiar.

A coleta de dados ocorreu por meio da análise dos prontuários e laudos dos pacientes que realizaram colonoscopias entre os meses de janeiro de 2003 a dezembro de 2007.

As variáveis avaliadas foram: idade (contínua em anos), sexo (masculino e feminino), presença de pólipos (sim e não), número de pólipos $(1,2,3,4,5,+$ de 5, e polipose adenomatosa), distribuição anatômica ao longo do intestino grosso (ceco, cólon ascendente, ângulo hepático, cólon transverso, ângulo esplênico, cólon sigmoide, reto e não informado), histologia do adenoma (tubular, túbulo-viloso e viloso), grau de displasia (alto grau, baixo grau, indeterminada), tipo de pólipo (pediculado, séssil e subpediculado) e tamanho $(<0,5 \mathrm{~cm}, 0,5 \mathrm{~cm}, 1 \mathrm{~cm}, 2 \mathrm{~cm}, 3 \mathrm{~cm}, 4 \mathrm{~cm},>5 \mathrm{~cm} \mathrm{e}$ não informado).

Para o critério de análise comparativa, os pacientes foram separados em grupos onde, na indicação do exame, constava a presença de história familiar para CCR e sem a presença de história familiar. Foi considerado paciente com história familiar positiva aquele possuidor de, ao menos, um familiar de primeiro grau com câncer colorretal.

Esses dois grupos foram divididos em subgrupos: pacientes com adenomas e presença de história familiar, e pacientes com adenomas, mas sem presença de história familiar para câncer colorretal, tendo como objetivo a análise comparativa entre eles.

O banco de dados foi criado através do aplicativo Excel 2007, sendo depois revisado com o objetivo de conferir sua exatidão. Posteriormente, os dados foram exportados, para outro programa, para análise 
estatística. Descreveram-se as variáveis categóricas, pela frequência absoluta e frequência relativa percentual, e as quantitativas, pela média e desvio padrão. Após, associaram-se as variáveis categóricas, pelo teste de qui-quadrado, e as quantitativas comparadas entre os grupos, pelo teste $t$ de Student, para amostras independentes. Foi considerado um nível de significância de 5\%.

Para a realização do estudo, remeteu-se o projeto ao Comitê de Ética em Pesquisa (CEP) do Hospital Nossa Senhora da Conceição, solicitando, junto ao diretor da instituição, autorização para o acesso aos dados já catalogados dos pacientes. Por se tratar de um estudo retrospectivo de revisão de prontuários, não foi aplicado o Termo de Consentimento Livre e Esclarecido (TCLE).

É importante ressaltar que todos os dados de identificação dos pacientes, obtidos no decorrer da análise dos laudos, foram mantidos em completo sigilo pela autora e por seu orientador.

\section{RESULTADOS}

O número total de pacientes submetidos à colonoscopia no período foi de 2.479; porém, 17 desses apresentaram polipose adenomatosa familiar sendo, portanto, excluídos do trabalho. Dessa forma, restou o total de 2.462 pacientes estudados.

Dos 2.462 pacientes analisados, 935 (38\%) eram do sexo masculino e 1.527 (62\%) do sexo feminino. A média de idade dos pacientes avaliados foi de 58,1 anos e a variação em torno da média foi de 15,5 anos. A idade mínima encontrada foi de um ano e a máxima foi de 98 anos.

Os pacientes foram divididos em dois grupos: pacientes em que a indicação do exame ocorreu por mo- tivo de história familiar positiva para câncer colorretal, totalizando $118(4,8 \%)$, sendo $27(22,9 \%)$ do sexo masculino e $91(77,1 \%)$ do sexo feminino. A média de idade deste grupo \pm desvio padrão foi de $54,4 \pm 13,5$ e, desses, apenas 18 (15,3\%) apresentaram adenomas. Pacientes que tiveram a indicação do exame associada a outros motivos, sem ser história familiar positiva para câncer colorretal, totalizaram 2.344 (95,2\%), sendo 908 (38,7\%) do sexo masculino e $1.436(61,3 \%)$ do sexo feminino. A média de idade \pm desvio padrão foi 58,4 $\pm 15,5$. Desses, $319(13,6 \%)$ apresentaram adenomas (Tabela 1).

Através do teste qui-quadrado de Pearson, com 5\% de significância, pode-se dizer que houve diferença estatisticamente significativa na comparação entre os sexos dos dois grupos como mostra a Tabela 1 $(\mathrm{P}=0,001)$. Pode-se também afirmar que houve diferença significativa na comparação entre as idades dos grupos ( $\mathrm{P}=0,002)$, ainda conforme a Tabela 1. Quanto à presença de adenomas, não houve diferença estatisticamente significativa entre eles $(\mathrm{P}=0,723)$.

Dentre os $18(15,3 \%)$ pacientes com adenomas e história familiar positiva para CCR, $4(22,2 \%)$ são do sexo masculino e $14(77,8 \%)$ do sexo feminino. A média de idade \pm desvio padrão desses 18 pacientes foi de $59,4 \pm 12,5$. Os achados colonoscópicos mostraram que $13(72,2 \%)$ pacientes apresentaram apenas um adenoma; 2 (11,1\%) apresentaram 2 adenomas; 2 (11,1\%) apresentaram 3 adenomas e $1(5,6 \%)$ paciente apresentou 5 adenomas, totalizando 28 adenomas. Entretanto, entre os $319(13,6 \%)$ pacientes com adenomas sem história familiar positiva para câncer colorretal, $163(51,1 \%)$ são do sexo masculino e $156(48,9 \%)$ são do sexo feminino. A média de idade \pm desvio padrão desse grupo foi de $62,6 \pm 14,6$. Os achados colonoscópicos evidenciam que 205 (64,3\%) apresentaram ape-

Tabela 1. Características dos pacientes que tiveram a indicação do exame de colonoscopia, motivada pela história familiar positiva para CCR, em comparação a pacientes sem história familiar positiva, com um total de 2.462 prontuários.

\begin{tabular}{|c|c|c|c|}
\hline Características gerais & $\begin{array}{c}\text { História familiar } \\
\mathrm{n}=118\end{array}$ & $\begin{array}{c}\begin{array}{c}\text { Sem história familiar } \\
\mathrm{n}=\mathbf{2 . 3 4 4}\end{array} \\
\end{array}$ & $\mathbf{p}$ \\
\hline \multirow{3}{*}{$\begin{array}{lc}\text { Sexo } & \text { Masculino } \\
\text { Média de idade } \pm \text { desvio padrão }\end{array}$} & $27(22,9 \%)$ & $908(38,7 \%)$ & \multirow{2}{*}{0,001} \\
\hline & $91(77,1 \%)$ & $1.436(61,3 \%)$ & \\
\hline & $54,4 \pm 13,5$ & $58,4 \pm 15,5$ & 0,002 \\
\hline Presença adenomas & $18(15,3 \%)$ & $319(13,6 \%)$ & 0,723 \\
\hline
\end{tabular}

Fonte: elaborado pelos autores. 
nas 1 adenoma; 65 (20,4\%) apresentaram 2 adenomas; $36(11,3 \%)$ tiveram 3 adenomas; $11(3,4 \%)$ apresentaram 4 adenomas e $2(0,6 \%)$ apresentaram 5 adenomas, totalizando 497 adenomas (Tabela 2).

Houve diferença estatisticamente significativa na comparação entre o sexo dos dois grupos, como mostra a Tabela $2(\mathrm{P}=0,032)$. Dessa forma, pode-se concluir que, nos pacientes com adenomas e com história familiar para CCR, o sexo feminino apresenta maior incidência de pólipos, em comparação aos pacientes do sexo masculino. Porém, nos pacientes com adenomas e sem história familiar para CCR, o sexo masculino apresentou incidência maior. Já, quanto a variável idade, o estudo não mostrou diferença $(\mathrm{P}=0,354)$, conforme mostrado na Tabela 2. Quanto ao número de adenomas, não houve diferença significativa entre os dois grupos $(\mathrm{P}=0,187)$ (Tabela 2$)$.

É importante ressaltar que dos 118 pacientes com história familiar positiva para CCR, 18 apresentaram adenomas; desses, 3 pacientes apresentaram concomitantemente pólipos hiperplásicos. Dos 2.359 pacientes sem história familiar positiva para CCR, 319 apresentaram adenomas; desses, 20 apresentaram simultaneamente pólipos hiperplásicos $(\mathrm{P}=0,178)$.

Foram comparados os adenomas dos pacientes com história familiar para CCR, com os adenomas dos pacientes sem história familiar. Analisando a distribuição anatômica dos mesmos, notou-se que não houve diferença significativa entre os grupos $(\mathrm{P}=0,067)$, sendo o segmento distal o mais acometido para am- bos. Quanto à implantação na mucosa, o tipo séssil foi o mais prevalente, e também não houve diferença significativa $(\mathrm{P}=0,435)$. Na variável tamanho, não se observou diferença entre os grupos $(\mathrm{P}=0,635)$, sendo os adenomas menores que $0,5 \mathrm{~cm}$ os mais comuns. Da mesma forma, com a variável conduta, não houve diferença significativa $(\mathrm{P}=0,649)$ e nota-se que a pinça hot-biopsy é a mais usada. A variável histológica mostra que, em ambos os grupos, a histologia tubular é a mais comum, sem diferença estatística $(\mathrm{P}=0,445)$ (Tabela 3).

Dos 28 adenomas dos pacientes com história familiar positiva para CCR, nenhum apresentou displasia de alto grau, $3(10,7 \%)$ apresentaram displasia indeterminada e $25(89,3 \%)$ pacientes apresentaram displasia de baixo grau. Entre os 319 adenomas dos pacientes sem história familiar positiva para CCR, 46 $(9,2 \%)$ apresentaram displasia de alto grau, $51(10,3 \%)$ apresentaram displasia indeterminada e $400(80,5 \%)$ mostraram displasia de baixo grau, não havendo assim, diferença estatisticamente significativa entre os grupos $(\mathrm{P}=0,240)$, conforme mostrado na Tabela 3 .

\section{DISCUSSÃO}

O CCR é uma doença comum e letal, influenciada por fatores genéticos e ambientais e pela interação entre ambos. A predisposição genética é um fator de risco dominante para alguns indivíduos; entretanto, fatores ambientais, incluindo dieta, exercí-

Tabela 2. Característica dos pacientes com adenomas e história familiar positiva para CCR, em comparação a pacientes com adenomas e sem presença de história familiar positiva para CCR.

\begin{tabular}{llccc}
\hline $\begin{array}{l}\text { Características } \\
\text { gerais }\end{array}$ & Masculino & $\begin{array}{c}\text { Adenomas com história } \\
\text { familiar } \\
\mathbf{n = 1 8}\end{array}$ & $\begin{array}{c}\text { Adenomas sem história } \\
\text { familiar } \\
\mathbf{n = 3 1 9}\end{array}$ & $\mathbf{p}$ \\
\hline Sexo & $\begin{array}{c}(22,2 \%) \\
14(77,8 \%)\end{array}$ & $163(51,1 \%)$ & 0,032 \\
Média de idade \pm & Feminino & $59,4 \pm 12,5$ & $62,6 \pm 14,6$ & 0,354 \\
desvio padrão & & & \\
Número adenomas & 1 & $13(72,2 \%)$ & $205(64,3 \%)$ & \\
& 2 & $2(11,1 \%)$ & $65(20,4 \%)$ & 0,187 \\
& 3 & $2(11,1 \%)$ & $36(11,3 \%)$ & \\
\hline
\end{tabular}

Fonte: elaborado pelos autores. 
cio físico, tabagismo e obesidade também são fatores de alto risco ${ }^{13,14}$.

A incidência do CCR varia muito, com as maiores taxas na América do Norte, Austrália e Europa. Os países em desenvolvimento têm taxas mais baixas, especialmente África e Ásia ${ }^{15}$. Essas diferenças geográficas parecem ser atribuíveis às diferenças nas exposições dietéticas e ambientais que são impostas sobre um fundo de susceptibilidade genética ${ }^{16}$.
Quanto à história familiar para CCR, este estudo evidenciou $118(4,8 \%)$ pacientes com história familiar positiva para CCR e $2.344(95,2 \%)$ sem história familiar. Nos Estados Unidos, $5 \%$ dos adultos (com idade entre 20 e 79 anos) têm um parente de primeiro ou segundo grau com CCR. Essa taxa aumenta com a idade, refletindo maior chance de desenvolver o câncer à medida que ficam mais velhos ${ }^{17}$.

Tabela 3. Características gerais dos adenomas de pacientes com história familiar positiva para CCR (28 pacientes), comparados com os adenomas de pacientes sem história familiar para CCR (497 pacientes).

\begin{tabular}{|c|c|c|c|c|c|c|}
\hline \multicolumn{2}{|c|}{ Características gerais } & \multicolumn{2}{|c|}{$\begin{array}{c}\text { Adenoma com história } \\
\text { familiar positiva } \\
\mathbf{n}=\mathbf{2 8}\end{array}$} & \multicolumn{2}{|c|}{$\begin{array}{c}\text { Adenoma com história } \\
\text { familiar negativa } \\
n=497\end{array}$} & $\mathbf{p}$ \\
\hline \multirow{7}{*}{ Local } & Ceco & 5 & 17,8 & 33 & 6,6 & 0,067 \\
\hline & C.ascendente & - & - & 70 & 14,2 & \\
\hline & A. hepático & 1 & 3,8 & 33 & 6,6 & \\
\hline & C. transverso & 5 & 17,8 & 78 & 15,7 & \\
\hline & A. esplênico & 1 & 3,8 & 9 & 1,8 & \\
\hline & C. descendente & - & - & 34 & 6,8 & \\
\hline & Distal* & 16 & 56,8 & 240 & 48,3 & \\
\hline \multirow[t]{3}{*}{ Tipo } & Pediculado & 4 & 14,3 & 104 & 21,0 & 0,435 \\
\hline & Séssil & 24 & 85,7 & 379 & 76,2 & \\
\hline & Subpediculado & - & - & 14 & 2,8 & \\
\hline \multirow[t]{7}{*}{ Tamanho } & $<0,5 \mathrm{~cm}$ & 18 & 64,3 & 303 & 61,0 & 0,635 \\
\hline & $0,5 \mathrm{~cm}$ & 8 & 28,6 & 92 & 18,5 & \\
\hline & $1 \mathrm{~cm}$ & 2 & 7,1 & 63 & 12,7 & \\
\hline & $2 \mathrm{~cm}$ & - & - & 21 & 4,2 & \\
\hline & $3 \mathrm{~cm}$ & - & - & 10 & 2,0 & \\
\hline & $4 \mathrm{~cm}$ & - & - & 3 & 0,6 & \\
\hline & $+5 \mathrm{~cm}$ & - & - & 5 & 1,0 & \\
\hline \multirow[t]{5}{*}{ Conduta } & Biopsiado & - & - & 14 & 2,8 & 0,649 \\
\hline & Pinça fria & 2 & 7,1 & 65 & 13,1 & \\
\hline & Hot-biopsy & 16 & 57,2 & 263 & 52,9 & \\
\hline & Alça polip.** & 10 & 35,7 & 146 & 29,4 & \\
\hline & Outra & - & - & 9 & 1,8 & \\
\hline \multirow[t]{3}{*}{ Histologia } & Tubular & 23 & 82,1 & 386 & 77,7 & 0,445 \\
\hline & Túbulo-viloso & 5 & 17,9 & 83 & 16,7 & \\
\hline & Viloso & - & - & 28 & 5,6 & \\
\hline \multirow[t]{3}{*}{ Displasia } & Alto grau & - & - & 46 & 9,2 & 0,240 \\
\hline & Indeterminada & 3 & 10,7 & 51 & 10,3 & \\
\hline & Baixo grau & 25 & 89,3 & 400 & 80,5 & \\
\hline
\end{tabular}

*Distal: reto + sigmoide; ** Polip: polipectomia.

Fonte: elaborado pelos autores. 
O risco de CCR está fortemente associado ao número e à idade dos parentes de primeiro grau com história de câncer colorretal. Cerca de 1,5 a 3\% dos pacientes submetidos à ressecção de um único CCR têm chance de, nos primeiros cinco anos pós-operatório, recidivar o tumor. Ter um único parente de primeiro grau afetado aumenta o risco em 1,7 vezes, em relação ao da população geral. O risco é ainda maior se parentes de primeiro grau desenvolveram câncer, ou se o diagnóstico da doença ocorreu com menos de 55 anos de idade ${ }^{18}$. Entretanto, uma avaliação mais aprofundada dos efeitos do grau de relação com o risco de desenvolver adenomas avançados ou até CCR deixa a desejar.

Este estudo não mostrou diferença significativa quanto à frequência de adenomas, comparando os pacientes com história familiar para CCR com os pacientes sem história familiar $(\mathrm{P}=0,723)$. Alguns estudos também verificaram que a frequência de adenomas foi semelhante em pacientes com parentes de primeiro grau com $\mathrm{CCR}$, em comparação a pacientes sem história familiar. A literatura mostra, no entanto, que adenomas avançados, ou seja, de alto grau, foram significativamente mais frequentes em pacientes com história familiar presente ${ }^{19}$. No nosso estudo a comparação de adenomas com displasia de alto grau não foi estatisticamente significativa.

A idade é um fator importante para o aparecimento de CCR esporádico. É raro o diagnóstico da doença antes de 40 anos de idade. Porém, a incidência começa a aumentar significativamente entre 40 e 50 anos, e a cada década seguinte, as taxas de incidência se tornam cada vez mais expressivas ${ }^{20}$. Os adenomas são encontrados em aproximadamente $25 \%$ das colonoscopias realizadas em pessoas com 50 anos, e em 50\% das pessoas com 70 anos $^{8}$. Nesta pesquisa, a média de idade \pm desvio padrão para os pacientes com história familiar para CCR foi de $54,4 \pm 13,5$, e para os pacientes sem história familiar foi de $58,4 \pm 15,5$, apresentando uma diferença significativa $(\mathrm{P}=0,002)$. Para os pacientes com adenomas e história familiar para CCR, a média de idade foi $59,4 \pm 12,5$, e para os pacientes com adenomas sem história familiar foi $62,6 \pm 14,6$, não mostrando diferença estatisticamente significativa entre essa variável $(\mathrm{P}=0,354)$. Esses dados demonstram que a presença de história familiar para CCR fez com que os pacientes se submetessem mais cedo a exame.
Porém, a comparação entre os pacientes possuidores de adenomas com história familiar e possuidores de adenomas sem história familiar mostrou idade semelhante. Dessa forma, pode-se concluir que a idade foi o fator de risco independente mais importante, em relação ao surgimento dos adenomas.

Quanto ao gênero, o presente estudo mostrou que, nos 118 pacientes com história familiar, a incidência foi maior no gênero feminino. Também nos 2.344 pacientes sem história familiar, evidenciou-se maior número entre mulheres $(\mathrm{P}=0,001)$. Na análise dos pacientes com adenomas e história familiar para CCR, também se observa maior incidência no gênero feminino. Em contrapartida, nos pacientes com adenomas sem história familiar houve maior incidência no gênero masculino $(\mathrm{P}=0,032)$. Estudos sugerem que os eventos iniciais dos adenomas são menos comuns no sexo feminino do que no masculino; no entanto, a progressão de um adenoma para um carcinoma é fortemente influenciada pelo sexo feminino ${ }^{21}$. Há estudos demonstrando um efeito protetor dos estrógenos na formação do CCR, e é possível que a progressão de um adenoma para adenocarcinoma seja acelerada em mulheres na pós-menopausa que não fazem reposição hormonal.

O risco de CCR aumenta conforme o tamanho do adenoma, ao número e a histologia ${ }^{22}$. Histologicamente, os adenomas podem ser classificados como componente viloso, túbulo-viloso ou tubular, sendo que o primeiro mostra uma tendência maior à displasia de alto grau. Todos os adenomas são considerados displásicos e, recentemente, a classificação da displasia reconhece dois tipos: alta e baixa, sendo que a alta mostra adenomas avançados ${ }^{3,6}$.

A literatura mostra que a histologia vilosa, o aumento progressivo do tamanho do pólipo e a displasia de alto grau são fatores de risco independentes para o câncer focal dentro de um adenoma individual. A maioria dos adenomas tem menos de um centímetro na colonoscopia ${ }^{8}$. A proporção de adenomas, com características histológicas avançadas (displasia de alto grau ou mais de $25 \%$ com hitologia vilosa), aumenta cerca de 1 a $2 \%$ em adenomas pequenos $(<5 \mathrm{~mm})$, de 7 a $12 \%$ em adenomas médios $(0,5$ a $1 \mathrm{~cm})$ e 20 a $30 \%$ em adenomas maiores de um centímetro ${ }^{23}$. As taxas de crescimento dos pólipos adenomatosos são variáveis e não seguem uma tendência linear consistente. A 
maioria dos pólipos pequenos exibe um crescimento mínimo ( $5 \mathrm{~mm} / \mathrm{ano})$. Estudos sugerem que a regressão completa é incomum ${ }^{24}$.

Em um relatório, por exemplo, pacientes com múltiplos adenomas grandes ou adenomas vilosos no retossigmoide tiveram um risco 6,6 vezes maior de malignidade subsequente, do que a população de referência. Outro relatório apontou que o tamanho dos pólipos $(>1 \mathrm{~cm})$ e adenomas localizados proximalmente eram independentes preditores de recorrência de adenomas de alto risco ${ }^{25}$. Um terceiro relatório mostrou que os pacientes com apenas 1 ou 2 adenomas tubulares menores do que um centímetro possuíam um baixo risco de neoplasia do cólon, após cinco anos de seguimento. A presença de adenomas múltiplos (mais de três), independentemente do tamanho, é um fator de risco para o câncer ${ }^{26}$.

A análise comparativa de pólipos de pacientes com história familiar e sem história familiar para CCR, neste estudo, mostrou que não houve associação estatisticamente significativa entre a variável história familiar e as variáveis local, tipo, tamanho, conduta, histologia e displasia. A comparação entre os adenomas mostra que, no grupo com história familiar, não houve nenhum adenoma com mais de um centímetro, nem do tipo viloso, e também não mostrou nenhum adenoma com displasia de alto grau, mesmo não sendo diferente estatisticamente do grupo sem história familiar para CCR. Esses dados chamaram a atenção de forma que, talvez, com uma amostra maior, seria possível demonstrar que o surgimento dos adenomas em pacientes com história familiar positiva ocorra em idade mais precoce.
$\mathrm{O}$ rastreamento de CCR exige um programa contínuo de testes adequados e com acompanhamento, pois a incidência de câncer colorretal continua a aumentar com a idade. O rastreio colonoscópico deve ser considerado, particularmente, quando existirem parentes de primeiro grau com CCR, se o aparecimento de câncer em algum membro na família for com 55 anos ou antes e, se há pacientes com história familiar de CCR em idade inferior a 40 anos. Assim, o rastreio deve começar aos 40 anos, ou 10 anos mais cedo do que o mais jovem a desenvolver cancer na família ${ }^{27}$.

\section{CONCLUSÃO}

A prevalência de adenomas entre os pacientes que realizaram colonoscopia, em que a indicação do exame foi devida à história familiar para $\mathrm{CCR}$, foi semelhante ao grupo sem história familiar $(\mathrm{P}=723)$.

O grupo com história familiar para CCR mostrou mais mulheres $(\mathrm{P}=0,001)$ e a idade também foi mais jovem $(\mathrm{P}=0,002)$.

A comparação entre o grupo com adenomas e história familiar para CCR mostrou um número maior de mulheres, que o grupo com adenomas sem história familiar para CCR $(\mathrm{P}=0,032)$.

Quando, neste estudo, os adenomas de pacientes com história familiar e sem história familiar para CCR foram analisados comparativamente, foi observado que não houve associação estatisticamente significativa entre a variável história familiar e as variáveis local, tipo, tamanho, conduta, histologia e displasia.

\footnotetext{
ABSTRACT: Colorectal cancer is a common and lethal disease, influenced by genetic and environmental factors and the interaction between both. The risk of colorectal cancer is strongly associated with the number and age of first degree relatives with colorectal cancer. Methods: A retrospective study was performed having as its objective all the patients submitted to colonoscopy in a reference hospital in the city of Porto Alegre, between 2003 and 2007. The goal is to study the prevalence of adenomas in patients with family history of colorectal cancer. Results: From the 2,462 analyzed patients, 118 had family history for colorectal cancer and 2,344 did not have. In both groups there was predominance of females $(\mathrm{P}=\mathbf{0 . 0 0 1})$. In the patients with adenomas and family history of colorectal cancer, there was predominance of females. In the patients with no family history of colorectal cancer there was predominance of males $(\mathrm{P}=\mathbf{0 . 0 3 2})$. The number of adenomas between them was not statistically significant $(\mathrm{P}=\mathbf{0 . 1 8 7})$. Conclusion: The group with family history of colorectal cancer has shown more women $(\mathrm{P}=\mathbf{0 . 0 0 1})$ and the age was also lower $(\mathrm{P}=0.002)$. The comparison between the group with adenomas and family history for colorectal cancer has shown a bigger number of women than the group with adenomas with no family history for colorectal cancer $(\mathrm{P}=\mathbf{0 . 0 3 2})$.
}

Keywords: adenoma; prevalence; colorectal cancer. 


\section{REFERÊNCIAS}

1. Jemal A, Siegel R, Ward E, Murray T, Xu J, Thun MJ. Cancer statistics, 2007. CA Cancer J Clin 2007;57(1):43-66.

2. Brasil. Ministério da Saúde. Instituto Nacional do Câncer. Incidência de câncer no Brasil 2007. [acesso em 2010 outubro 3]. Disponível em: http://www.inca.gov.br.

3. Rossi BM, Nagawawa WT, Ferreira FO, Aguiar Jr. S, Lopes A. Classificação morfológica de pólipos colorretais. In: Nakagava W, Ferreira F. Câncer de cólon, reto e ânus. São Paulo: Lemar e Tecmed; 2004.

4. Bodger K. Colorectal cancer. J R Coll Physicians Lond 2000;34:197-201.

5. Vijan S, Inadomi J, Hayward RA, Hofer TP, Fendrick AM. Profections of demand and capacity for colonoscopy related to increasing rates of colorectal cancer screening in the United States. Aliment Pharmacol Ther 2004;20(5):507-15.

6. Miller KM, Waye JD. Approach to colon polyps in the elderly. Am J Gastroenterol 2000;95(5):1147-51.

7. Bond JH. Interference with the adenoma-carcinoma sequence. Eur J Cancer 1995;31A(7-8):1115-7.

8. O'Brien MJ, Winawer SJ, Zauber AG, Gottlieb LS, Sternberg SS, Diaz B, et al. The National Polyp Study: patient and polyp characteristics associated with high-grade dysplasia in colorectal adenomas. Gastroenterology 1990;98(2):371-9.

9. Cutait R, Borges JLA, Costa F. Câncer Colorretal. In: Mincis M. Gastroenterologia e hepatologia. Diagnóstico e tratamento. São Paulo: Lemos Editorial \& Gráficos Ltda; 2002.

10. Bonardi RA, Sartor MC, Baldin JA, Nicollelli GM, Duda JR, Olandoski M. História familial e câncer colorretal em idade precoce: deve-se indicar colectomia estendida? Rev bras Coloproctol 2006;26(3):244-8.

11. Zarchy TM, Ershoff D. Risk of colorectal cancer in families of patients with adenomatous polyps. N Engl J Med 1996;334(20):1339-40.

12. Wegener M, Borsh G, Shimidt G. Colorectal adenomas distribution, incidence of malignant transformation, and rate of recurrence. Dis colon rectum 1986;29(6):383-7.

13. Little J, Faivre J. Family history, metabolic gene polymorphism, diet and risk of colorectal cancer. Eur J Cancer Prev 1999;8(Suppl 1):S61-72.

14. Potter JD. Colorectal cancer: molecules and populations. J Natl Cancer Inst 1999;91(11):916-32.

15. Parkin DM, Pisani P, Ferlay J. Global cancer statistics. CA Cancer J Clin 1999;49(1):33-64.

16. Ashktorab H, Nouraie M, Hosseinkhah F, Lee E, Rotimi C, Smoot D. A 50-year review of colorectal cancer in African
Americans: implications for prevention and treatment. Dig Dis Sci 2009;54(9):1985-90.

17. Ramsey SD, Yoon P, Moonesinghe R, Khoury MJ. Populationbased study of the prevalence of family history of cancer: implications for cancer screening and prevention. Genet Med 2006;8(9):571-5.

18. Atkin WS, Morson BC, Cuzick J. Long-term risk of colorectal cancer after excision of rectosigmoid adenomas. N Engl J Med 1992;326(10):658-62.

19. Lindgren G, Liljegren A, Jaramillo E, Rubio C, Lindblom A. Adenoma prevalence and cancer risk in familial non-polyposis colorectal cancer. Gut 2002;50(2): 228-34.

20. Eddy DM. Screening for colorectal cancer. Ann Intern Med 1990;113(5):373-84.

21. Crandall CJ. Estrogen replacement therapy and colon cancer: a clinical review. J Womens Health Gend Based Med 1999;8:1155-1166.

22. Laiyemo AO, Murphy G, Albert PS, Sansbury LB, Wang Z, Cross AJ, et al. Postpolypectomy colonoscopy surveillance guidelines: predictive accuracy for advanced adenoma at 4 year. Ann Intern Med 2008;148(6):419-26.

23. Lieberman D, Moravec M, Holub J, Michaels L, Eisen G. Polyp size and advanced histology in patients undergoing colonoscopy screening: implications for CT colonography. Gastroenterology 2008;135(4):1100-5.

24. Bersentes K, Fennerty MB, Sampliner RE, Garewal HS. Lack of spontaneous regression of tubular adenomas in two years of follow-up. Am J Gastroenterol. 1997;92(7): 1117-20.

25. Martinez ME, Sampliner R, Marshall Jr, Bhattacharyya AK, Reid ME, Alberts DS. Adenoma characteristics as risk factors for recurrence of advanced adenomas. Gastroenterology 2001;120(5):1077-83.

26. Lieberman DA, Weiss DG, Harford WV, Ahnen DJ, Provenzale D, Sontag SJ, et al. Five-year colon surveillance after screening colonoscopy. Gastroenterology 2007;133(4): 1077-85.

27. Burt RW, DiSario JA, Cannon-Albright L. Genetics of colon cancer: Impact of inheritance on colon cancer risk. Annu Rev Med 1995;46:371-9.

\section{Endereço para correspondência:}

Bianca Zandoná

Rua Padre Hildebrando 585/409 - São João

CEP: 91030-310 - Porto Alegre (RS), Brasil

E-mail: biancazandona@yahoo.com.br 